\title{
Successful healing treatment of high blood cholesterol levels and asthma using Yoga Prana Vidya (YPV) system: A case study of self-healing
}

\author{
V S Nanduri ${ }^{1 *}$, Alok Vasavda ${ }^{2}$ \\ ${ }^{1}$ Consultant, ${ }^{2}$ YPV Healer, Yoga Prana Vidya Ashram, Research, Thally, Tamil Nadu, India
}

*Corresponding Author: V S Nanduri

Email: vsnanduri@yahoo.com

\begin{abstract}
Introduction: A 33 year old male had been suffering from Asthma since childhood and he was diagnosed with high cholesterol after he grew up. He has a strong family history of Cardiac Disease with high blood Cholesterol. The prescribed medications for asthma did not help him much. He did not take any medication for Cholesterol. Being a Yoga Prana Vidya (YPV) trained Healer he used self-healing protocols.

Materials and Methods: This is case study method with detailed analysis of medical records of the patient's health conditions pre and post YPV healing treatment applied as alternative medicine.

Results: Analysis of the patient's health condition and medical case documents pre and post treatment show positive improvement obtained with YPV healing method for treating high blood cholesterol and Asthma.

Conclusion: This case study documents the evidence gathered on the effective use of YPV in self-healing and treating High Blood Cholesterol and Asthma. To produce holistic and optimum results, YPV uses a combination of approaches that include: Physical exercises, rhythmic yogic breathing, Salt free diet and fruit diet, Méditation techniques, healing by trained and experienced healers, participation in group healing, regular self-practice of specified techniques. There is ample scope to conduct further research on the application of YPV as alternatively effective and low cost medicine for various medical conditions.
\end{abstract}

Keywords: Asthma, Breathing Difficulty, Breathlessness, High Blood Cholesterol, LDL, HDL, TCH, Yoga Prana Vidya (YPV) Healing.

\section{Introduction}

Coronary heart disease (CHD) is one of the major causes of death in United States for both men and postmenopausal women in western world. A study conducted in India suggests that the prevalence of CHD is about $10 \%{ }^{1}$ Dyslipidemia is an abnormal amount of lipids (e.g. triglycerides, cholesterol and/or fat phospholipids) in the blood. ${ }^{2}$ In developed countries, most dyslipidemias are hyperlipidemias; that is, an elevation of lipids in the blood. This is often due to diet and lifestyle. Dyslipidemia is one of the important modifiable risk factors in CHD.

Cholesterol is a fatty substance which is made in the liver. It's found in some foods too. All need some cholesterol in their bodies just to keep ticking over but having too much can clog up arteries and lead to health problems in the future. ${ }^{5}$ It is a waxy substance found in blood. Human body needs cholesterol to build healthy cells, but high levels of cholesterol can increase risk of heart disease. With high cholesterol fatty deposits can develop in blood vessels. Eventually, these deposits grow, making it difficult for enough blood to flow through the arteries. Sometimes, those deposits can break suddenly and form a clot that causes a heart attack or stroke. High cholesterol can be inherited, but it's often the result of unhealthy lifestyle choices, which make it preventable and treatable. A healthy diet, regular exercise and sometimes medication can help reduce high cholesterol. ${ }^{6}$

Asthma is a lung disease that makes it harder to move air in and out of the lungs. Asthma is chronic and one has to live with it every day. It can be serious, even life-threatening. There is no cure for asthma, but it can be managed so one can live a normal, healthy life. With asthma, the airways in lungs are often swollen or inflamed. This makes them extra sensitive to things that one is exposed to in the environment every day, or asthma "triggers." A trigger could be a cold or the weather, or things in the environment, such as dust, chemicals, smoke and pet dander. ${ }^{7}$ Asthma is a common chronic inflammatory disorder affecting about 300 million people worldwide. As a holistic therapy, yoga has the potential to relieve both the physical and psychological suffering of people with asthma, and its popularity has expanded globally. ${ }^{3}$ However, according to the authors, a number of clinical trials have been carried out to evaluate the effects of yoga practice, with inconsistent results. ${ }^{3}$

\section{Yoga Prana Vidya System (YPV)}

Patients with common diseases such as cholesterol and Asthma often face a very unfortunate and worrisome journey from diagnosis to treatment. The reality of common diseases is that since a lot of people have them, many take them for granted. Experience has shown that it is only when the common diseases become critical, the patients start thinking of acting upon corrective actions. ${ }^{4}$ Common diseases can also become dangerous, because at times some medical doctors even may not see them. Just by knowing the name of the disease and correlating the basic symptoms as a due diligence, the doctors conclude the prescription and medical line of action. One such example is of high cholesterol in patients. It has been observed that Yoga Prana Vidya ${ }^{\mathrm{TM}}$, (YPV) ${ }^{\circledR}$, which is a no-touch, no-drug, bio-energy healing method, has been used successfully to heal patients having simple to complex and multiple medical conditions. This paper presents one case study of a patient having a high blood cholesterol and Asthma issue, successfully healed by $\mathrm{YPV}^{\odot}$.

There are many recorded medical cases which show that YPV healing system has been successfully applied in management and control of various medical conditions such 
as:

1. Diabetes

2. Blood pressure and heart ailments

3. ENT and respiratory ailments

4. Musculoskeletal

5. First aid and emergency cases

6. Dental cases

7. Eye camps

8. Cancer cases

9. Digestive disorders

10. Psychological cases like anxiety, depression, suicidal tendencies, relationship issues, lack of attention and concentration

11. Criminal tendencies etc.

Some recent publications of noteworthy cases showing efficacy of YPV healing have appeared in peer reviewed journals cited in references. ${ }^{1,8-11}$

The concept of Yoga has to be viewed and understood in a broader and deeper sense as elucidated by the founder of the Yoga Prana Vidya System. ${ }^{12}$ Yoga in recent times is used to refer to Asanas or postures of physical exercises and sometimes even to pranayama or a form of systematic breathing exercise. These are in fact parts of Yoga and not the complete Yoga. If viewed from the Yogi Patanjali point of view, Yoga is actually known as Ashtanga Yoga which means the Yoga consisting of 8 steps. There are many levels of Yoga in practice. Each of the steps is very important.

As per the ancient Yogic teachings, it is the Soul using this physical body, the physical body being an important vehicle of the Soul. This vehicle is extremely important in the physical world and it must be maintained, sustained and used for the purpose of the Divine. The purpose of yoga is to achieve the union with the Divine and manifest our own greatness on Earth.

The Soul (referring here to the Incarnated Soul or the Jeevatma) has 3 vehicles through which it operates:

1. The Energy Body known as Pranamaya Kosha, including Physical body known as Annamaya Kosha.

2. The Emotional Body or the Kamamaya Kosha

3. The Mental Body or the Manomaya Kosha

Considering the above, it is deduced that to sustain, maintain and/or regain good health, it is not adequate to treat just the Physical Body. It also involves maintaining a healthy state of one's Energy Body. This is because our Energy or the Etheric Body is the basic mould of the physical body, which science now recognises as a bio-plasmic body. Anything that happens to the physical body, happens to the Etheric Body. The Etheric Body is therefore referred to as "Etheric Double" in his theosophy literature. ${ }^{13}$ Any ailment in the physical body has its effect on the Etheric Body. This is also a reason, why we use the term "Prana Vidya". Prana is Life Force or Energy used for the maintenance of the energy body and Vidya is the technology of how to do so. The technology of maintaining the energy body is therefore termed Yoga Prana Vidya (or YPV) system which offers techniques for maintaining the physical and energy body and also maintain the etheric connectivity with the higher self.

Healing is the process by which the energy body can be renewed thus bringing change in the physical body, because the former interpenetrates the latter. Used up energy or diseased energy can be removed and the energy body can be impregnated with fresh energy. All biological life on earth has the ability to heal itself. Energy follows thought and energy accelerates the healing process. So the energy is used in healing, to accelerate the healing process and the body heals itself. Healing involves 2 steps: Cleansing, or removing the used up energy and Energizing, or giving fresh energy.

The air around us contains a life force that keeps us alive. This life force is also known as "Prana" or the "Breath of Life". YPV reveals various techniques of using prana in order to promote good health. Yoga Prana Vidya has been used as a tool to deal with psychological and emotional issues also to remedy everyday work and financial stresses of life. It is primarily used as a no-touch, no-drug, healing system, complementary (along with) with other healing modalities like Allopathy, Ayurveda, and Homeopathy. However, there are numerous instances where the application of YPV healing alone has been sufficient to alleviate the ailment/disease (both physical and psychological).

In YPV, the healer becomes a channel of energy who receives and transfers energy. Yoga Prana Vidya (YPV) is a revolutionary form of energy "medicine". It is an ancient science and art that has been revived in a new form which is easily adaptable and in tune with modern day busy life. There are two modes of YPV healing. In one mode, a healer heals other persons. In the other mode, a healer can heal him/herself which is known as self-healing. ${ }^{12}$

In this paper, the authors are presenting a self-healing case, where the healer applied YPV Healing on himself to treat high cholesterol and Asthma over a period of about six months. In view of the persistent YPV self-healing and application of associated techniques, the patient got healed completely without any need of any form of medical intervention as detailed in the following sections.

\section{Case Report}

\section{Patient's background}

A 33 years old male, residing in Ahmedabad City, Gujarat State, India. Qualified with a Master's degree, professional accountancy and working as a corporate executive, married and has one child.

\section{Pre - YPV medical history}

The patient was diagnosed to be suffering with childhood Asthma from childhood, which surfaced first at the age of 6 years. Also, he was diagnosed to be having a high level of blood cholesterol since the age of 18 years. The patient has a strong family history of having high blood cholesterol and associated cardiac ailments. The patient regularly had blood tests (once in a year) to monitor blood parameters, especially cholesterol. Looking at the rising trend and the strong family history, the consulting cardio physician had recommended medical intervention to bring the blood parameters to normal range. The patient was guided that once the parameters are 
normalised with medical treatment, he could gradually reduce drug dependency and maintain normal level of cholesterol through dietary discipline and regular physical exercises.

In the matter of Asthma, in general the period between November and February months tended to be very difficult for the patient, since the Asthma gets triggered most easily during these months. For the rest of the year, Asthma gets triggered only if rigorous exercise is done or if any kind of stress is experienced.

\section{Medical Treatment Recommended Before YPV}

The patient was advised to take ATORVASTATIN for 6 months. Based on how well the drug responds, the patient was supposed to modify the dosage and once the level of Cholesterol comes back to normal range, he would have been advised gradual reduction in dose. Due to inner resistance, he never took medicines. Knowing that this was just borderline high, he avoided medication. Additionally, the patient was advised to have food with less oil and minimise junk food. This he could follow quite easily, since general food preparation at home had less oil and he was not much used to junk food habits.

The Total Cholesterol Level of the patient usually remained in a range of 225 to $235 \mathrm{mg} / \mathrm{dl}$. The test conducted on 07 December 2018 confirms this and shows a level of $229 \mathrm{mg} / \mathrm{dl}$ [See Annexure Fig. 1: Report before YPV Healing].

\section{Asthma Condition and medications before YPV}

He had Asthma since the age of six. Doctors then told that this is a childhood Asthma and should get healed naturally by the age of 12 or 13 years. This did not happen, in fact, the Asthma issue increased. His Asthma issue was at its peak while he was around 15 years old. The winter months especially between October and February - were the toughest months for him. After Diwali festival, when the air pollution levels are high, he used to start feeling breathlessness at night time. The typical trigger points were:

1. Rigorous exercises (including playing cricket for 15 minutes or more)

2. Speaking continuously for 45 minutes or more (including reading paragraphs from the school text book for such period)

3. Eating ice cream or drinking cold beverages (including cold water)

4. Using "cotton-stuffed" bed or cushions (instead of foam based bed or cushion)

5. Eating full stomach meals at night and trying to sleep within 1 hour of dinner

At the age of 15, his parents approached a Homeopathic practitioner who prescribed some pills that happened to be very effective. After few months, they coincidently discovered that these pills contained steroids and they immediately stopped the treatment as advised by another doctor. Later he was advised to use Asthalin inhaler on SOS basis and take Deriphyllin and Asthalin $4 \mathrm{mg}$ tablets. He used to have the tablets regularly, however, before sleeping use of the inhaler was mandatory, without which he could not sleep. Medical opinion indicated that this was more of a psychological instinct and not a requirement of the body to take the inhaler, and facing the breathlessness is a different matter. However, the patient knew that the issue was not psychological, but quite real. This condition remained the same for over a decade and in time, use of the inhaler had become as frequent as having water in between during the day.

\section{YPV Intervention}

In December 2018, when he got the opportunity to join the One Year Spiritual Intensive Program at the YPV Ashram, his only concern was that the climate was cooler and more humid at the Ashram compared to the city. This could trigger Asthma very easily. Also, the beds and cushions at the Ashram were cotton-stuffed, being another major trigger point.

The patient had been a practitioner of YPV holistic integrative healing system since many years. He had undertaken many simple to critical healing cases for numerous people who had requested healing. Around early December 2018, he decided to work on himself and bring his health parameters to normal, including the Asthma and High Blood Cholesterol issue.

The patient followed the following YPV protocols.

1. Rhythmic Yogic breathing, 3 times a day (as guided in YPV Sadhana App) ${ }^{12}$

2. Forgiveness Sadhana 2 times daily (as guided in YPV Sadhana App)

3. Planetary peace meditation once a day (including Super brain asana in the morning and physical exercise before and after meditation) - as guided in YPV Sadhana App.

4. Salt-free diet

5. Healing once a day for 1 month (December 2018 January 2019), and once a week for 6 months. (19 March 2019 and 18 September 2019.

\section{Results}

Consequent to the above stated YPV healing treatment, the results are as stated below.

\section{Cholesterol}

The Total Cholesterol Level came down to $206 \mathrm{mg} / \mathrm{dl}$ by 04 January 2019 [Annexure, Fig. 2 - Report during middle of YPV Healing] and $147 \mathrm{mg} / \mathrm{dl}$ by 24 September 2019 [Annexure, Fig. 3 - Report after YPV Healing].

\section{Status of Asthma condition after YPV healing}

He applied self-healing protocols upon himself for the entire month of December 2018 and later once a week from March 2019. No symptom of breathing difficulty experienced throughout the period of healing. The patient stated that, "With tremendous gratitude and with utmost honesty I can say that I have not taken a single dose of the inhaler, nor a single tablet for Asthma!" As a part of the programme schedule, he participated in physical exercises for about 90 minutes in a day, walking/running for about 15 minutes in a 
day and breathing exercises for about 40 minutes in a day. He would not have been able to do any of these under the Asthma conditions before healing.

\section{Effects on the other parameters}

Table 1 shows some physical parameters which are normalized as a result of holistic aspect of YPV healing, and also blood pressure measurements.

Table 1: Patient's physical parameters

\begin{tabular}{|l|c|c|}
\hline & Pre-YPV & Post -YPV \\
\hline Height, cm & 168 & 168 \\
\hline Weight. Kg & 75 & 54 \\
\hline Waist, inches & 34 & 28 \\
\hline BMI & 26.57 & 19.1 \\
\hline Blood Pressure & $115 / 89$ & $100 / 80$ \\
& $(19 / 03 / 2019)$ & $(19 / 09 / 2019)$ \\
\hline
\end{tabular}

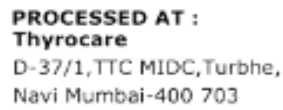

Corporate Office : Thyrocare Technologies Limited P D-37/3, TTC MIDC, Turbhe, Navi Mumbai - 400703 (c) $022-30900000 / 41252525 \approx 8691866066 \approx$ wellness @thyrocare.com Cwww.thyrocare.com

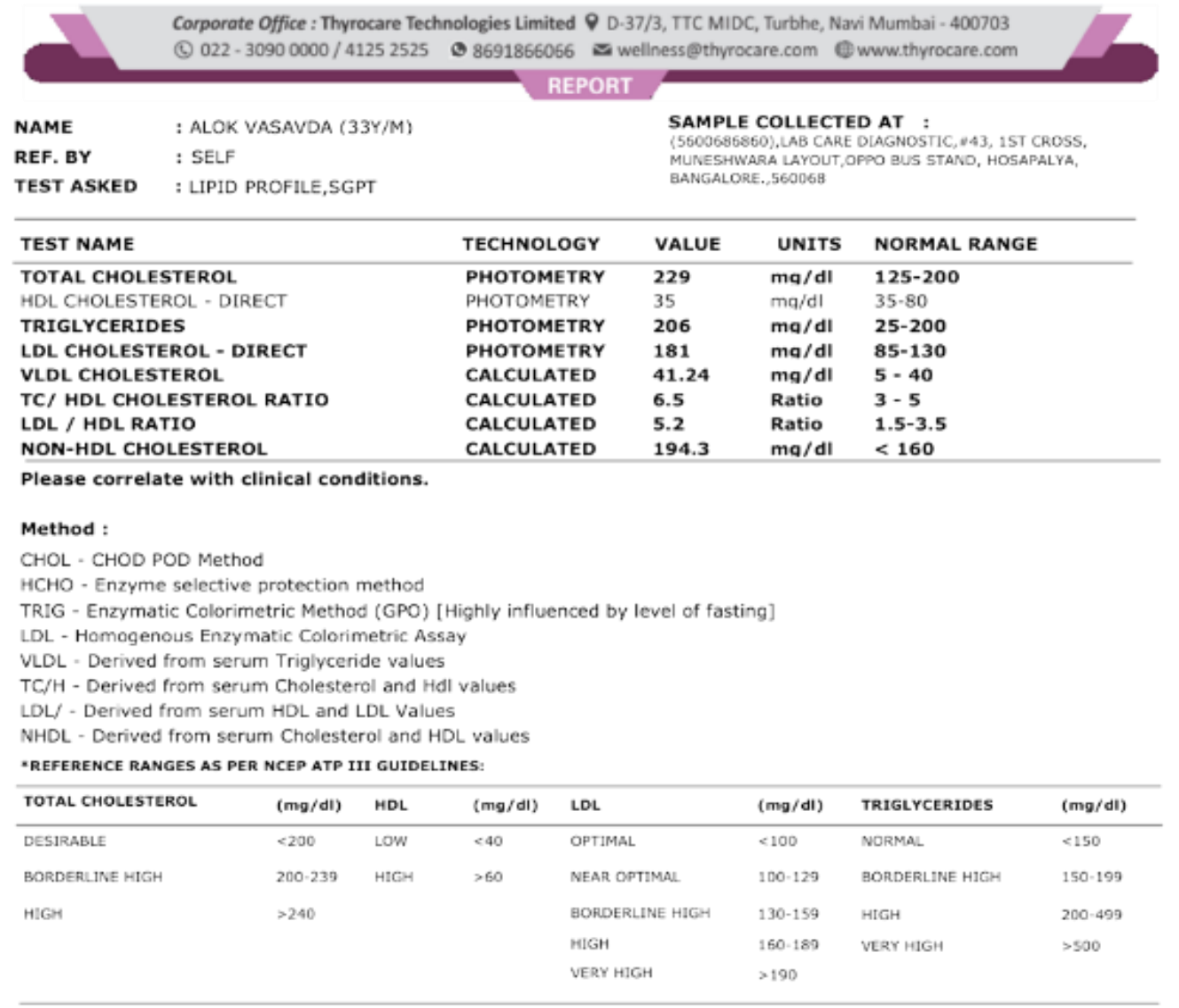

Alert III 10-12 hours fasting is mandatory for lipid parameters. If not, values might fluctuate.

\begin{tabular}{ll}
\hline Sample Collected on (SCT) & $: 07$ Dec 2018 09:00 \\
Sample Received on (SRT) & $: 08$ Dec 2018 02:47 \\
Report Released on (RRT) & $: 08$ Dec 2018 04:39 \\
Sample Type & $:$ SERUM \\
Labcode & $: 071244568 / A 9345$ \\
Barcode & $:$ M0929893
\end{tabular}

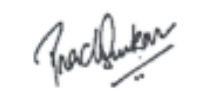

Dr.Prachi Sinkar MD(Path)

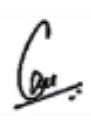

Dr. Caesar Sengupta MD(Micro) Page : 1 of 4

Fig. 1: Report before YPV Healing, dated 8 December, 2018 
PROCESSED AT :

Thyrocare

Samanvitha Complex, No.12,13 and 14,

Mayura Street, Outer Ring Road,

Hebbal, Banqalore-560095

Think Thyroid. Think Thyrocare.

Corporate Office : Thyrocare Technologies Limited P D-37/3, TTC MIDC, Turbhe, Navi Mumbai - 400703

(4) $022-30900000 / 4125252588691866066 \approx$ wellness@thyrocare.com Owwwthyrocare.com

NAME : ALOK VASAVDA $(33 Y / M)$

REF. BY : SELF

TEST ASKED : LIPID PROFILE
SAMPLE COLLECTED AT :

(5600688143),LABCARE DIAGNOSTIC HSR, M39 MANGAMANAPALYA SLV ROAD NEAR VENKASHWERA TEMPEL. BENGLORE 560068,560068

\begin{tabular}{|c|c|c|c|c|}
\hline TEST NAME & TECHNOLOGY & VALUE & UNITS & NORMAL RANGE \\
\hline TOTAL CHOLESTEROL & PHOTOMETRY & 206 & ma/dl & $125-200$ \\
\hline HDL CHOLESTEROL - DIRECT & PHOTOMETRY & 34 & $\mathrm{ma} / \mathrm{dl}$ & $35-80$ \\
\hline TRIGLYCERIDES & PHOTOMETRY & 139 & $\mathrm{ma} / \mathrm{dl}$ & $25-200$ \\
\hline LDL CHOLESTEROL - DIRECT & PHOTOMETRY & 143 & $\mathrm{ma} / \mathrm{dl}$ & $85-130$ \\
\hline VLDL CHOLESTEROL & CALCULATED & 27.78 & $\mathrm{mq} / \mathrm{dl}$ & $5-40$ \\
\hline TC/ HDL CHOLESTEROL RATIO & CALCULATED & 6 & Ratio & $3-5$ \\
\hline LDL / HDL RATIO & CALCULATED & 4.2 & Ratio & $1.5-3.5$ \\
\hline NON-HDL CHOLESTEROL & CALCULATED & 171.72 & $\mathrm{ma} / \mathrm{dl}$ & $<160$ \\
\hline
\end{tabular}

Please correlate with clinical conditions.

Method :

CHOL - CHOD POD Method

$\mathrm{HCHO}$ - Enzyme selective protection method

TRIG - Enzymatic Colorimetric Method (GPO) [Highly influenced by level of fasting]

LDL - Homogenous Enzymatic Colorimetric Assay

VLDL - Derived from serum Triglyceride values

$\mathrm{TC} / \mathrm{H}$ - Derived from serum Cholesterol and $\mathrm{Hdl}$ values

LDL/ - Derived from serum HDL and LDL Values

NHDL - Derived from serum Cholesterol and HDL values

-REFERENCE RANGes AS PER NCEP ATP III GUIDELINES:

\begin{tabular}{|c|c|c|c|c|c|c|c|}
\hline TOTAL CHOLESTEROL & (mg/dI) & HDL & $(\mathrm{mg} / \mathrm{dl})$ & LOL & (mg/dl) & TRIGLYCERIDES & $(\mathrm{mg} / \mathrm{dl})$ \\
\hline DESIRABLE & $<200$ & Low & $<40$ & OPTIMAL & $<100$ & NORMAL & $<150$ \\
\hline BORDERLINE HIGH & $200-239$ & $\mathrm{HIGH}$ & $>60$ & NEAR OPTIMAL & $100-129$ & BORDERLINE HIGH & 150-199 \\
\hline \multirow[t]{3}{*}{ HIGH } & $>240$ & & & BORDERLINE HIGH & $130-159$ & HIGH & $200-499$ \\
\hline & & & & $\mathrm{HIGH}$ & 160-189 & VERY HIGH & $>500$ \\
\hline & & & & VERY HIGH & $>190$ & & \\
\hline
\end{tabular}

Alert III 10-12 hours fasting is mandatory for lipid parameters. If not, values might fluctuate.

$\sim$ End of report

\begin{tabular}{ll}
\hline Sample Collected on (SCT) & $: 04$ Jan 2019 09:00 \\
Sample Received on (SRT) & $: 04$ Jan $201916: 10$ \\
Report Released on (RRT) & $: 04$ Jan 2019 18:52 \\
Sample Type & $:$ SERUM \\
Labcode & Barcode
\end{tabular}

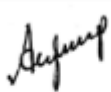

Dr Arjun CP MD(Path)

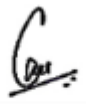

Dr.Caesar Sengupta MD(Micro) Page : 1 of 2

Fig. 2: Report midway during YPV Healing Intervention 


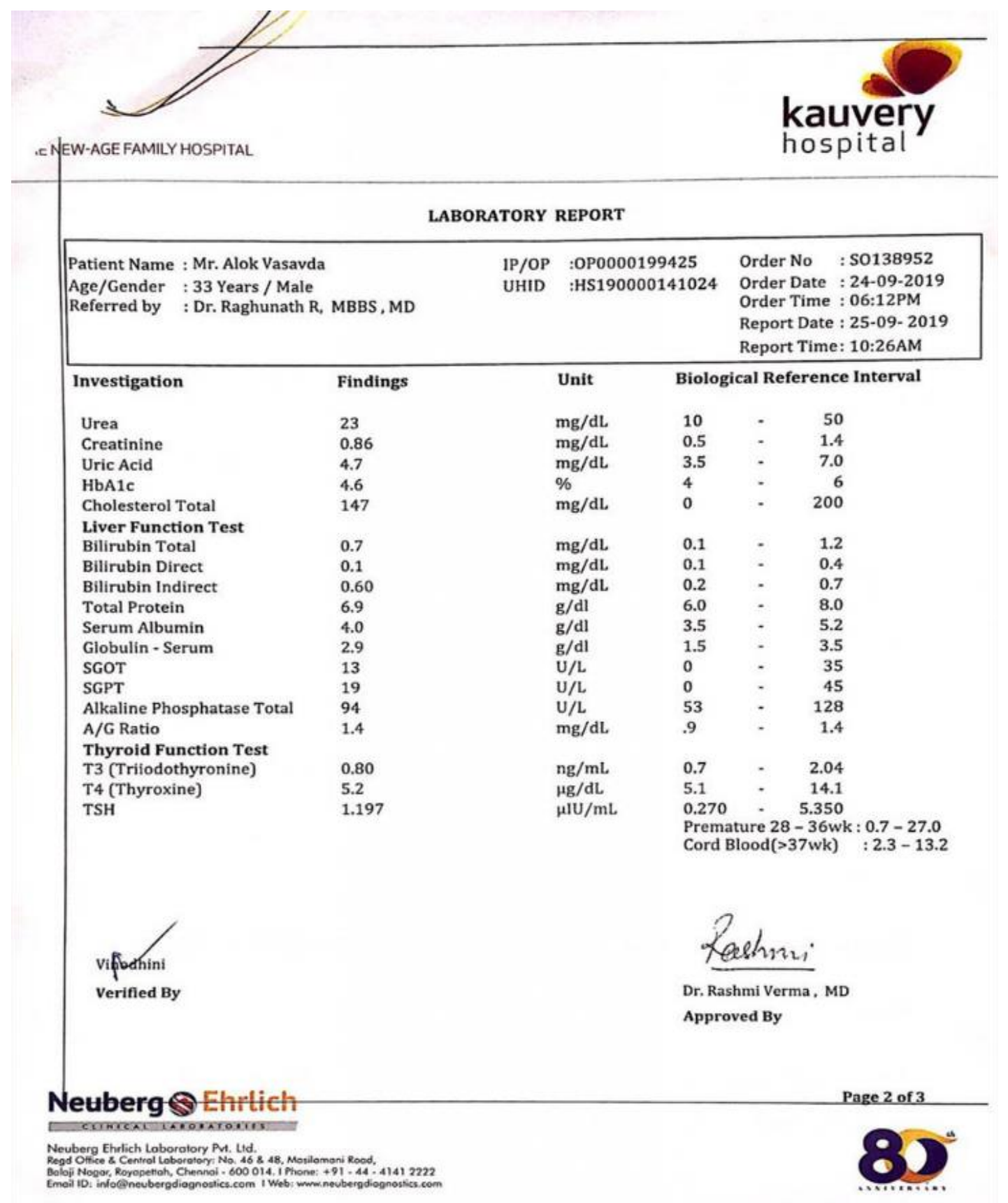

Fig. 3: Report after YPV Healing completed

\section{Discussion}

In this case study, it was observed that by application of YPV self-healing and slight changes in life style, the Cholesterol levels came down to normal range. Also, the breathlessness and Asthma have been healed completely. YPV healing is a no-drug healing modality, the patient could successfully overcome his high Cholesterol and Asthma ailments without any need of medications. At the present time, the patient is leading an extremely healthy life, full of vitality, beyond his expectations.

It is noted from the research literature that a study by Nisha Shanthakumari et al. (2013) shows that Yoga, being a lifestyle modifier incorporating exercise and stress management training, targets the elevated lipid through integrated approaches resulting in improved lipid profiles, lower BMI, and macro-vascular complications in diabetes. ${ }^{14}$ This case study supports similar findings using several variants of Yoga applications in health improvements. For example a systematic review by Paula Chu et al (2014) revealed that there is promising evidence of yoga on improving cardio-metabolic health, showed significant improvements for cholesterol, blood pressure, body weight and BMI. ${ }^{15}$

It is further observed that the Asthma condition of the patient also greatly improved. This outcome supports a study by Mekonnen and Mossie (2010) using a variant of Yoga that reported statistically significant reduction in day and night 
asthma attacks in the yoga group. ${ }^{16}$ Another relevant study, Yoga for Asthma, (Yang et al, 2016) supports this case with the conclusion that exercise, yogic breathing and meditation have positive effects on the quality of life for Asthma patients. $^{3}$

It is further seen in this study (Table 1) that the physical parameters such as weight and BMI have also got normalised as a result of the holistic nature of YPV healing and associated techniques. This finding is similar to the findings on holistic healing results of YPV reported earlier in the literature references. ${ }^{4,8-11}$

\section{Conclusion}

This case report documents the evidence gathered on the effective use of YPV in healing high blood Cholesterol and Asthma. The general conditions under which YPV produces optimum results is also derived from the fact that YPV uses a combination of approaches, such as: (a) Physical exercises including rhythmic yogic breathing, (b) Salt free diet and fruit diet, (c) Meditation techniques, (d) Healing by trained and experienced healers, (e) Patient's participation in group healing, and (f) Regular self-practice of specified Sadhanas.

YPV has been helping as a preventive medicine, when the seekers are receptive and undertake regular practices of the self-practice techniques of YPV such as exercises, Rhythmic breathing, meditation and salt free controlled diet.

\section{Acknowledgements}

The authors express sincere thanks to Sri Ramana Trust for giving permission to use their copyright terms, Yoga Prana Vidya $^{\mathrm{TM}}$ and $\mathrm{YPV}^{\odot}$, and also for allowing them to use the patient medical reports and data in this report.

\section{Source of funding}

None.

\section{Conflict of interest}

None.

\section{References}

1. K.V.V. Prasad, Madhavi Sunita, P. Sitarama Raju, M. Venkata Reddy, B.K. Sahay, K. J. R. Murthy. Impact of pranayama and yoga on lipid profile in normal healthy volunteers. $J$ Exercise Physiol online, 2006;9(1):1-6.

2. Wikipedia. Dyslipidemia. Available [online] https://en.wikipedia.org > wiki > Dyslipidemia

3. Yang ZY, Zhong HB, Mao C, Yuan JQ, Huang YF, Wu XY, Gao YM, and Tang JL. Yoga for Asthma. Cochrane Database Syst Rev 2016;4:CD010346. doi: 10.1002/14651858.CD010346.pub2. Available [Online] https://www.ncbi.nlm.nih.gov/pubmed/27115477

4. Jayachander Reddy Neravetla, Venkata Nanduri. A study into successful treatment of some difficult Medical cases using Yoga Prana Vidya (YPV) Healing System as alternative medicine. Int J Sci Eng Res 2019;10(7):882-7.

5. Heart UK. What is Cholesterol? Available [online] https://www.heartuk.org.uk/cholesterol/what-is-cholesterol

6. Mayo Clinic. High Cholesterol. [Online] Available https://www.mayoclinic.org/diseases-conditions/high-bloodcholesterol/symptoms-causes/syc-20350800
7. American Lung Association. What is Asthma. Available [Online] https://www.lung.org/lung-health-and-diseases/lungdisease-lookup/asthma/learn-about-asthma/what-isasthma.html

8. Ashalatha HR, Ramya Ashwin and Venkata Nanduri. Diabetes Management and Control Using Yoga Prana Vidya (YPV) Healing System. J Biol Life Sci, 2019;10(2):106-20.

9. Ramya Ashwin, Venkata Satyanarayana Nanduri. Cardiac Case Study: Successful Healing Treatment of a 48-Year-Old Male with Block in Heart, Using Yoga Prana Vidya (YPV) Healing System. Saudi J Nurs Health Care 2019;2(11):353-6.

10. Rajkumari Sachdeva \& Venkata Satyanarayana Nanduri. Management of Post-Herpetic Neuralgia PHN) by Yoga Prana Vidya (YPV) Healing: A Case Study. Am J Biomed Life Sci 2019;7(6):174-8.

11. Gupta K, \& Nanduri VS. A case report of the Exostosis of ear of an elderly female: Successful healing with Integrated Yoga Prana Vidya (YPV) healing approach as alternative to surgical intervention. Ann Geriatrics Educ Med Sci 2019;6(2):42-7.

12. Yoga Prana Vidya Ashram. YouTube channel. [Online] Available https://www.youtube.com/channel/UChDXESyqOblNP3LUTuyUfw/videos

13. Powell A E. The Etheric Double: The Health Aura of Man. Wheaton IL (USA): Quest Books (Revised edition January 1, 1997).

14. Nisha Shantakumari, Shiefa Sequeira, and Rasha El deeba. Effects of a yoga intervention on lipid profiles of diabetes patients with dyslipidemia. Indian Heart J 2013;65(2):127-31. doi: 10.1016/j.ihj.2013.02.010

15. Paula Chu, Rinske A Gotink, Gloria Y Yeh, Sue J Goldie, MG Myriam Hunink First. The effectiveness of yoga in modifying risk factors for cardiovascular disease and metabolic syndrome: A systematic review and meta-analysis of randomized con-trolled trials. Eur J Prev Cardiol 2016;23(3):291-307. Article first published online: December 15, 2014; Issue published: February 1, 2016. https://doi.org/10.1177/2047487314562741

16. Demeke Mekonnen, Andualem Mossie. Clinical Effects of Yoga on Asthmatic Patients: A Preliminary Clinical Trial. Ethiop J Health Sci 2010;20(2):107-12. 